MS23 Charge and spin density

of materials at extreme

conditions
3 C. P. Brock et al., Acta Cryst. B, 1991, 47, 789-797

4 V. Pichon-Pesme et al., J. Phys. Chem, 1995, 99, 6242-6250

5 M. Milaneso et al., J Mol Struct-TheoChem, 1997, 419, $139-154$

6 B. Dittrich et al., Angew. Chem. Int. Ed. Engl., 2004, 43, 2718-2721

Keywords: charge density,

Chairs: Nicola Casati, Philippe Guionneau

\section{MS23-P1 Vitamin C Revisited}

Natalie T. Johnson ${ }^{1}$, Michael R. Probert ${ }^{1}$

1. Department of Chemistry, Newcastle University, Newcastle Upon Tyne, UK

email: N.Johnson5@newcastle.ac.uk

X-ray charge density (CD) studies are notorious for requiring high quality diffraction data to ensure the production of physically meaningful results. With continual improvements in both processing software and experimental hardware it may be useful to revisit previously studied compounds. This may afford the option to fully test these developments to discover additional subtleties in the structures. Alongside this, there is growing research into the reproducibility of CD refinements that have continued beyond the investigations into oxalic acid dihydrate ${ }^{1}$. This original study found that for four $C D$ studies while significant features were reproducible in electron density maps, both 'peak shapes and locations of maxima differ in detail', leading researchers to advise caution when interpreting experimental distributions.

Further concern was raised when discrepancies were found between the dipole moment calculated across half of 2,2'-(1,4-Phenylene)dimalononitrile ${ }^{2}$. When varying either the instrument or refinement procedure the calculated dipoles ranged from $0.84 \mathrm{D}$ to $5.36 \mathrm{D}$. It is imperative that $\mathrm{CD}$ studies, as with other studies, be reproducible in order to be able to confidently draw conclusions about the electron density of molecules. However, it has previously been shown that the CD multipole parameters of chemical fragments of molecules are transferable between systems where the fragments are in similar environments ${ }^{3-4}$.

The availability of high quality crystals of vitamin $\mathrm{C}$ provide an ideal test case for our preliminary work into $\mathrm{CD}$ reproducibility. The $\mathrm{CD}$ of vitamin $\mathrm{C}$ has previously been studied at $120 \mathrm{~K}^{5}$ and refined to give good agreement with theoretical and neutron studies. New data will be presented along with an exploration of the reproducibility of charge density models.

An additional property of vitamin $\mathrm{C}$ that is of interest is that it crystallizes with $Z^{\prime}>1$ which further allows the investigation into the transferability of multipole parameters between chemical moieties, an underlying requirement for the use of non spherical parameters in models derived from standard resolution datasets ${ }^{6}$.

1 P. Coppens et al., Acta Cryst. A, 1984, 40, 184-195

2 M. R. Probert, private communication, 2007 